\title{
Charge Assisted Laser Desorption/Ionization Mass Spectrometry of Droplets
}

\author{
Kaveh Jorabchi, Michael S. Westphall, and Lloyd M. Smith \\ Department of Chemistry, University of Wisconsin-Madison, Madison, Wisconsin, USA
}

We propose and evaluate a new mechanism to account for analyte ion signal enhancement in ultraviolet-laser desorption mass spectrometry of droplets in the presence of corona ions. Our new insights are based on timing control of corona ion production, laser desorption, and peptide ion extraction achieved by a novel pulsed corona apparatus. We demonstrate that droplet charging rather than gas-phase ion-neutral reactions is the major contributor to analyte ion generation from an electrically isolated droplet. Implications of the new mechanism, termed charge assisted laser desorption/ionization (CALDI), are discussed and contrasted with those of the laser desorption atmospheric pressure chemical ionization method (LDAPCI). It is also demonstrated that analyte ion generation in CALDI occurs with external electric fields about one order of magnitude lower than those needed for atmospheric pressure matrix assisted laser desorption/ionization or electrospray ionization of droplets. (J Am Soc Mass Spectrom 2008, 19, 833-840) @ 2008 American Society for Mass Spectrometry

$\mathrm{M}$ ass spectrometry (MS) has attracted great attention in recent years, partly because of its capabilities in life science applications. Hyphenated techniques with MS, e.g., HPLC-MS, have become the workhorse of biochemical and biological research in areas such as proteomics. This new era of mass spectrometry started with two Nobel prize winning methods, electrospray ionization (ESI) and matrix assisted laser desorption/ionization (MALDI), to produce gas-phase ions of proteins and peptides [1, 2]. Although the instrumentation for separating and detecting the gas-phase ions has advanced significantly since then, little work has been done to address and alleviate the intrinsic limitations of ESI and MALDI. This is partly because whereas mass spectrometer designs are based on well-understood electrostatic and electrodynamic processes, the fundamentals of gas-phase ion generation are still relatively poorly understood.

Currently, mass spectrometry suffers from two major problems: (1) ion suppression effects and (2) difficulties in quantification. These problems severely limit the application of MS in the analysis of mixtures, and both are rooted in ionization. The efficiency of gasphase ion generation with current techniques depends heavily on the analyte's chemical structure. Further, the amount of charge available for ionization is limited in the current methods, creating a competition for charge between the analytes in mixtures. With such shortcomings, the ionization step may be considered the weakest link and the bottleneck in mass spectrometry at its current stage. Therefore, more vigorous research has

Address reprint requests to Professor L. M. Smith, Department of Chemistry, University of Wisconsin-Madison, 1101 University Avenue, Madison, WI 53705, USA. E-mail: smith@chem.wisc.edu been devoted to the study of ion generation in recent years, resulting in several new ionization techniques such as desorption electrospray ionization (DESI) [3], atmospheric pressure photoionization (APPI) [4], laser desorption atmospheric pressure chemical ionization (LD-APCI) [5-7], electrospray assisted laser desorption ionization (ELDI) [8, 9], laser ablation electrospray ionization (LAESI) [10], no-discharge APCI [11, 12], direct analysis in real time (DART) [13], desorption atmospheric pressure chemical ionization (DAPCI) [14], laser-induced acoustic desorption (LIAD) [15, 16], field-induced droplet ionization (FIDI) [17], and nanostructure-initiator ionization [18]. Among the techniques cited, APPI, ELDI, LAESI, LD-APCI, and LIAD are similar in the sense that they utilize a two-step approach in which desorption and ionization occur during two separate physical processes. Such an approach is expected to minimize ion suppression effects by providing more control over each step. Utilization of a corona discharge (e.g., in LD-APCI) to supply the charge for ionization is particularly advantageous because of the quantitative control on the extent of the charge produced by this method (current monitoring). This study is focused on understanding the role of corona discharge in laser desorption mass spectrometry of droplets and liquid samples, with the ultimate goal of improving ionization performance.

Droplets and liquid matrices provide unique advantages over solid samples for laser desorption MS techniques [19-21]. A liquid surface represents an ideal homogenous sample for laser desorption because the concentrations of analyte and matrix are constant across the sample. Further, the self-healing property of the liquid renews the surface between laser shots, allowing prolonged analysis without need to raster the sample 
surface with the laser beam. This eliminates the laser spot dependence of the analytical signal and improves reproducibility. In addition, the stable ion beam from liquid samples allows better instrument optimization leading to higher quality data. For example, excellent calibration curve linearity $\left(R^{2}>0.995\right)$ over three orders of magnitude along with acceptable precision (RSD < $10 \%$ ) has been demonstrated for peptide analysis in both vacuum and atmospheric pressure MALDI using liquid matrices [7, 21].

In this report, we investigate corona-induced enhancement of peptide ion signal using laser desorption from droplets. Pulsed corona discharge along with time-resolved UV-laser desorption and ion extraction is used to gain insight into the mechanism of ion generation. Finally, we describe a new ionization technique, termed charge-assisted laser desorption/ionization (CALDI), based on the insights gained.

\section{Experimental}

Experiments were performed using a droplet deposited on a 1/16-in.-diameter steel or Teflon support rod located $2 \mathrm{~mm}$ from the extended nozzle of an ESI-TOF instrument (Mariner; Applied Biosystems, Foster City, CA). A stainless steel needle located $6 \mathrm{~mm}$ from the extended nozzle and centered with the nozzle opening supplied the corona ions. The relative positioning of the droplet, laser optics, and corona needle with respect to the mass spectrometer inlet is shown in the lower section of Figure 1. Two modes of operation were studied: (1) pulsed mode and (2) continuous mode. In the pulsed mode, a ring electrode (placed $4 \mathrm{~mm}$ from the nozzle) and timing electronics were employed to allow timecontrolled generation of corona ions (Figure 1). In the continuous mode, however, the corona discharge was on at all times and no ring electrode/timing electronics were used. Stainless steel was used as the support material for experiments where a potential was directly

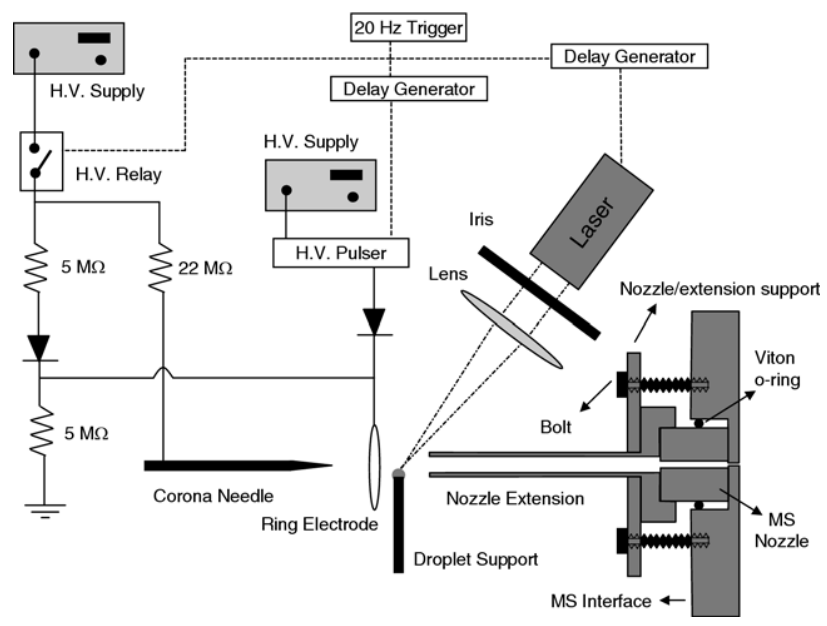

Figure 1. Experimental apparatus for pulsed corona ion production, laser desorption, and ion extraction. applied to the droplet. In other experiments where electrical isolation of the droplet was required, a Teflon support was employed. Details of the instrumentation for the two modes along with the sample preparation procedure are described below.

The nozzle extension was a stainless steel tube $(5 \mathrm{~cm}$ long, 2-mm i.d.) designed to alleviate the spatial constraints and to facilitate the positioning of the droplet and laser optics. The original nozzle was pressed into an opening in the base of the extension tube, providing electrical/thermal contact and a vacuum seal. The new nozzle assembly was held in place by a stainless steel support bolted to the MS interface. The nozzle and extension were held at $65 \mathrm{~V}$ using an external power supply. In addition, a 1-M $\Omega$ resistor provided an electrical path to ground from the nozzle/extension, preventing charge build-up on the nozzle and protecting the MS internal electronics in the unlikely event of an arc from the corona needle to the nozzle.

A spherical fused silica lens (1-in. diameter, 50-mm focal distance; Edmund Optics, Barrington, NJ) focused 337-nm laser light (NL100 nitrogen laser; Stanford Research Systems Inc., Sunnyvale, CA) onto the surface of the droplet closest to the MS inlet. The laser output power was adjusted by high voltage potentiometer on the laser and an adjustable iris placed in the laser beam path before the lens. The laser repetition rate in all experiments was $20 \mathrm{~Hz}$. The positive corona discharge voltage $(+4.5 \mathrm{kV})$ was provided by a Bertan power supply (Series 205B) and an additional power supply (model PS350; Stanford Research Systems Inc., Sunnyvale, CA) applied a potential of $+2 \mathrm{kV}$ to the droplet support in the experiments where a stainless steel support was used (continuous mode).

Pulsed experiments were performed using a Teflon droplet support. A ring electrode was inserted between the corona needle and the droplet in these experiments to provide an electric field independent of the corona needle (see Figure 1). This allowed establishment of an electric field in the droplet area even when the corona voltage was off. The succession of events in the pulsed mode was triggered at $20 \mathrm{~Hz}$ by a pulse generator (PM5715, Philips). The trigger pulse actuated the high voltage relay, applying proper voltages to the needle and the ring electrode and resulting in production of positive corona ions throughout the duration of the trigger pulse. A secondary voltage was then applied to the ring electrode using a delayed pulse generator (TGP110; Tenma, Centerville, $\mathrm{OH}$ ), which in turn triggered a high voltage pulser (PVX-4140; Directed Energy Inc., Fort Collins, $\mathrm{CO}$ ) connected to a high voltage power supply. An additional delayed pulse generator (TGP110, Tenma) controlled the timing of the laser shot. To monitor the sequence of the pulses, an analog oscilloscope (2465 B; Tektronix, Beaverton, OR) was employed. It was triggered by the main $20-\mathrm{Hz}$ trigger signal; channel one of the scope measured the ring electrode voltage using a high voltage probe (P5100, 
Table 1. Comparison of MRFA signal-to-background and signal-to-noise ratios for three ionization methods ${ }^{\text {a }}$

\begin{tabular}{|c|c|c|c|}
\hline \multicolumn{2}{|c|}{ Operating parameters } & $\frac{\mathrm{S} / \mathrm{B}}{5}$ & $\frac{\mathrm{S} / \mathrm{N}}{14}$ \\
\hline $\begin{array}{l}\text { LD-APCl conditions: } \\
\text { - Sample on a stainless steel support at }+2 \mathrm{kV} \\
\text { - Low laser fluence ( } 4 \mu \mathrm{J} / \mathrm{pulse}, 0.017 \mathrm{~mm}^{2} \text { area) } \\
\text { - Average of } 10 \text { spectra ( } 3 \mathrm{~s} \text { integration each) }\end{array}$ & $\begin{array}{l}3 \mathrm{kV} \text { sharp needle (no corona ions) } \\
4.5 \mathrm{kV} \text { sharp needle (corona ions, } 3 \mu \mathrm{A} \text { ) } \\
4.5 \mathrm{kV} \text { Blunt probe (no corona ions) }\end{array}$ & $\begin{array}{r}5 \\
9 \\
10\end{array}$ & $\begin{array}{l}14 \\
28 \\
43\end{array}$ \\
\hline $\begin{array}{l}\text { Electrically isolated droplet conditions }\left(\mathrm{CALDI}^{\mathrm{b}}\right) \text { : } \\
\text { - Sample on a Teflon support } \\
\text { - High laser fluence }\left(20 \mu \mathrm{J} / \text { pulse, } 0.026 \mathrm{~mm}^{2} \text { area) }\right. \\
\text { - Average of } 10 \text { spectra ( } 3 \mathrm{~s} \text { integration each) }\end{array}$ & $\begin{array}{l}4.5 \mathrm{kV} \text { sharp needle (Corona ions, } 3 \mu \mathrm{A} \text { ) } \\
4.5 \mathrm{kV} \text { Blunt probe (No corona ions) }\end{array}$ & $\begin{array}{l}66 \\
\text { N.D. }\end{array}$ & $\begin{array}{l}238 \\
\text { N.D. }\end{array}$ \\
\hline $\begin{array}{l}\text { Standard AP-MALDI: } \\
\text { - Sample on a stainless steel support at }+2 \mathrm{kV} \\
\text { - High laser fluence ( } 20 \mu \mathrm{J} / \mathrm{pulse}, 0.026 \mathrm{~mm}^{2} \text { area) } \\
\text { - Average of } 10 \text { spectra ( } 3 \mathrm{~s} \text { integration each) }\end{array}$ & $\begin{array}{l}\text { Stainless steel post is inline with nozzle } \\
\text { 2-mm sample nozzle distance }\end{array}$ & 66 & 281 \\
\hline
\end{tabular}

a1- $\mu \mathrm{L}$ droplet of $100 \mu \mathrm{M}$ concentration is used for all the experiments. Data calculated based on peak heights.

${ }^{b}$ Charge assisted laser desorption/ionization; see text for discussion of this mechanism.

${ }^{\mathrm{c}}$ Not detected.

Tektronix) and channel two monitored the firing of the laser using the synchronous output of the laser.

The pulsing electronics described above allows independent timing control of three events: (1) positive corona ion production, (2) electric field establishment in the droplet area, and (3) laser firing. This capability permits experiments to be performed that provide valuable insight into the mechanism of corona-enhanced analyte ion signal generation in laser desorption from droplets.

The droplets in these experiments were composed of a mixture of a liquid matrix and analyte solutions. The liquid matrix was prepared based on a formulation developed for AP-liquid MALDI [22]. In this formulation, a liquid support is prepared by mixing 1:1 volumetric ratios of diethanolamine and $50 \%$ ethanol. $\alpha$-Cyano-4-hydroxycinnamic acid is then dissolved in the liquid support to a concentration of $600 \mathrm{mM}$. The resulting liquid matrix is then sonicated for $15 \mathrm{~min}$ and vortexed for $1 \mathrm{~min}$ before use. Peptides MRFA (MetArg-Phe-Ala, Average MW = 523.7) and bradykinin (Arg-Pro-Pro-Gly-Phe-Ser-Pro-Phe-Arg, Average MW = 1060.2) were dissolved in $0.1 \%$ formic acid to yield 3 $\mathrm{nmol} / \mu \mathrm{L}$ and $1 \mathrm{nmol} / \mu \mathrm{L}$ stock solutions, respectively. For analysis, peptide stock solutions were diluted with the matrix solution to yield a concentration of $100 \mu \mathrm{M}$ for each peptide. A 1- $\mu \mathrm{L}$ droplet of this analyte solution was then placed on the droplet support. HPLC-grade water was purchased from J. T. Baker and 200-proof ethanol was from Pharmaco-Aaper (Brookefield, CT). All other materials were purchased from Sigma-Aldrich (St. Louis, MO) and used with no further purification.

\section{Results and Discussion}

\section{Continuous Operating Mode}

The experimental apparatus for the continuous corona mode (no ring electrode, corona on at all times) is similar to that employed in ultraviolet (UV) LD-APCI studies by Turney and Harrison [7]. We tested the generation of corona discharge ions using a corona tip held at $+4.5 \mathrm{kV}$ and a stainless steel droplet support at $+2 \mathrm{kV}$. In this configuration, a current of $3 \mu \mathrm{A}$ is registered by the high voltage (HV) power supply and positive corona ions are detected in the mass spectra. A $2-\mu \mathrm{L}$ droplet of the matrix and analyte mixture was then placed on the support and the laser intensity was adjusted to provide attenuated AP-MALDI signal at low laser fluence $\left(4 \mu \mathrm{J} /\right.$ pulse, $0.017 \mathrm{~mm}^{2}$ laser spot area). In accord with the observations of Turney and Harrison, a significant enhancement of the peptide ion signals was observed when the corona discharge was on compared to when the corona was turned off (Table 1, LD-APCI data). This effect has been attributed to the postdesorption ionization of neutrals by corona ions [7]. However, the effect of the corona needle on the electric field that drives the ions to the MS inlet was not fully evaluated in the previous studies. To evaluate this effect, we replaced the corona needle with a blunt stainless steel probe of the same o.d. and applied the same potential as to the corona needle. In this configuration, no current is registered by the HV power supply and no corona ions are detected by the MS. Thus, although the blunt probe provides approximately the same electric field as the corona needle, it does not result in the production of corona ions. Interestingly, we observed a similar enhancement in the peptide signals with the high voltage applied to the blunt probe (Table 1, LD-APCI data), indicating that the electric field rather than gas-phase neutral ionization is the major factor in the signal enhancement. Note that UV-LD-APCI provides little analytical use in terms of sensitivity because it requires operation in the lowfluence regime [7].

A far more interesting phenomenon was observed when the stainless steel droplet support was replaced with a Teflon support and high fluence laser desorption was utilized. In this case, Teflon electrically isolates the 


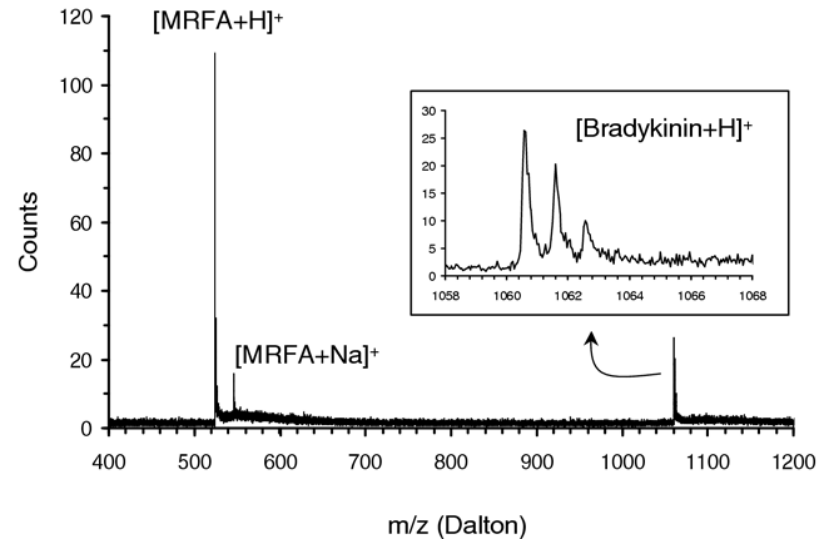

Figure 2. Mass spectra of MRFA $(100 \mu \mathrm{M})$ and bradykinin (100 $\mu \mathrm{M})$ in continuous mode using Teflon support and corona needle at $+4.5 \mathrm{kV}(3 \mu \mathrm{A})$. The desorption is effected at high laser fluence $\left(770 \mathrm{~J} / \mathrm{m}^{2}\right)$ and the spectrum is obtained by averaging 10 spectra collected at $3 \mathrm{~s} /$ spectrum.

droplet and no direct potential is applied to the sample. With the application of $+4.5 \mathrm{kV}$ to the corona needle, corona ions were generated and peptide ion signals were observed in the mass spectra upon laser desorption (Figure 2). However, when the blunt probe was used at the same potential (no corona ions) with constant laser desorption parameters, no peptide ion signals were detected. Table 1 summarizes these observations under electrically isolated droplet conditions. The effects observed with the Teflon support are clearly related to the absence and presence of corona ions and at first seem contrary to the conclusions achieved above using a stainless steel droplet support. However, as we will show in the next section by the pulsed experiments, this phenomenon can be explained based on a new mechanism, termed charge assisted laser desorption ionization (CALDI). As indicated in Table 1, CALDI provides similar sensitivity to that of standard liquid AP-MALDI. Both methods are operated at high laser fluence compared to UV-LD-APCI. Further, no significant improvement in analytical performance for APMALDI and CALDI was observed in our experiments at fluences above $800 \mathrm{~J} / \mathrm{m}^{2}$. Both methods also provide similar signal stability ( $\sim 10 \%$ RSD based on 10 spectra with $3 \mathrm{~s}$ integration time per spectrum).

\section{Pulsed Operation Mode}

Two hypotheses may be considered to explain the peptide signal enhancement by corona ions using a Teflon support: (1) secondary ionization of gas-phase neutrals produced by laser desorption and (2) interaction of corona ions with the droplet, leading to laser desorption from a charged droplet. These two scenarios can be distinguished by the dependence of the analyte ion signal on the timing of the laser pulse relative to the timing of corona ion production.

For gas-phase neutral ionization to occur, the corona ions must overlap with the gas-phase neutrals (gener- ated by the laser pulse) in space and time; i.e., to observe the peptide ions, laser desorption should occur while the corona ions are in the droplet vicinity. Therefore, a strong dependence of peptide ion signal on the relative timing of corona ion production and laser desorption is expected for the gas-phase neutral ionization mechanism. In contrast, for the charged droplet mechanism, the extent of analyte ion generation should be largely independent of the timing of corona ion production relative to laser desorption. In other words, peptide signals should be observed in this case as long as the droplet becomes charged before the laser shot. The pulsed corona experiments described below were aimed at revealing the dominant mechanism of signal enhancement induced by corona ions for an electrically isolated droplet.

The sequence of events is depicted in Figure 3 for one cycle at $20 \mathrm{~Hz}$. Each cycle is divided into four periods. In Period I, corona ions are generated by applying 4.5 $\mathrm{kV}$ to the corona needle through the high voltage relay along with a simultaneous supply of $2.25 \mathrm{kV}$ to the ring electrode via the voltage divider. Then the system is allowed to rest in Period II during which the ring electrode is set to $0 \mathrm{~V}$ and no potential is applied to the corona needle (HV relay is open). In Period III, the HV pulser applies $2.25 \mathrm{kV}$ to the ring electrode, establishing an electric field in the droplet area. This period is used to study the effect of the electric field on peptide ion signals in the absence of gas-phase corona ions. During Period IV the system is again at rest as in Period II. The laser is independently controlled by a delay generator and can be fired during any of the four periods.

The effect of the laser shot timing on analyte ion signal is illustrated in Figure 4. The data in Figure 4 clearly demonstrates that analyte ion generation is enhanced only when the laser is fired in Periods I and III. In Period I, the corona ions are present around the droplet when the desorption occurs; however, in Period III, the corona ions are removed from the droplet vicinity by the electric field before desorption. Note that

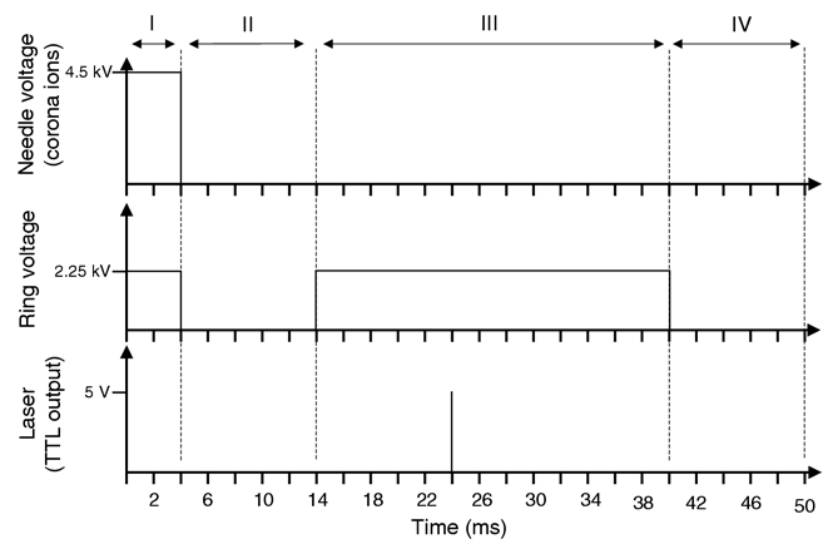

Figure 3. Timing diagram of pulsed corona ion generation, ion extraction electric field (ring electrode), and laser desorption for one cycle at $20 \mathrm{~Hz}$. The laser can be fired at any time during the cycle. 


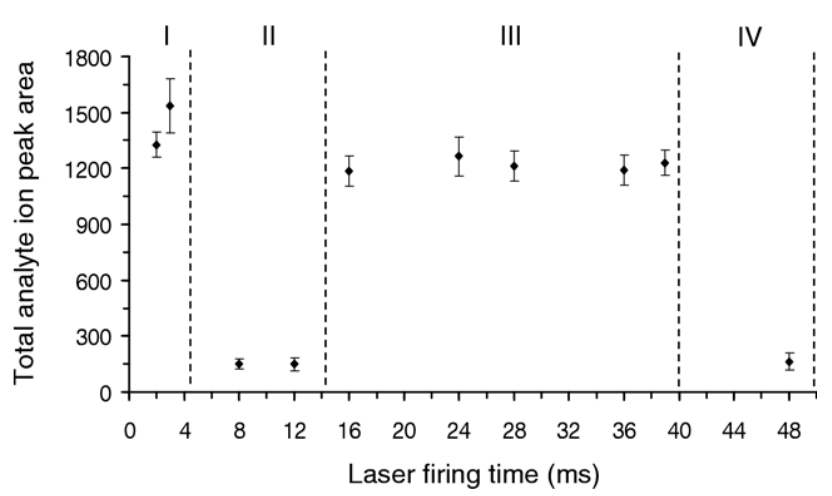

Figure 4. Sum of analyte ion peak areas for $[\mathrm{MRFA}+\mathrm{H}]^{+}$and [bradykinin $+\mathrm{H}]^{+}$as a function of laser shot timing (see Figure 3 ). Each point is from an average of 10 mass spectra acquired at $3 \mathrm{~s}$ per spectrum. The error bars reflect the standard deviation for the 10 measurements.

the analyte ions generated by the laser shot at $39 \mathrm{~ms}(1$ $\mathrm{ms}$ before the electric field shut down in Period III) are collected by the MS with an efficiency similar to all the other firing events in Period III. This shows that the analyte ions at the droplet surface require less than $1 \mathrm{~ms}$ to travel out of the droplet area and to the MS inlet under the electric field established in Period III. As the ion mobilities of small corona ions are greater than or equal to those of the larger analyte ions, one can conclude that the corona ion density is reduced dramatically around the droplet by the electric field and the ions are eventually cleared out of the droplet area in early stages of Period III $(<5 \mathrm{~ms}$ to be conservative considering the 4-mm distance between the ring and the MS inlet). Observation of similar peptide ion intensity throughout Periods I and III along with the dramatic change in corona ion density across Period III compared with Period I indicates that analyte ion generation is independent of the presence/density of corona ions at the desorption moment, strongly suggesting that gasphase corona ion-neutral reactions do not play a significant role in ionization. Another possibility for explaining the relatively flat signal intensity throughout Period III is that the neutrals generated in this period remain in the droplet area and become ionized by corona ions in Period I of the next cycle. However, this mechanism would lead to signal enhancement in Periods II and IV similar to Period III. The experimental data in Figure 4 shows clearly that this does not occur, eliminating the possibility of such gas-phase ion-neutral reactions. Therefore, peptide ion signal enhancement in this case cannot be attributed to the chemical ionization mechanism that occurs via corona ions in APCI.

Although the possibility of gas-phase ion-neutral reactions was eliminated, it is noteworthy that the corona ions are necessary to observe the peptide ion signal for an electrically isolated droplet (see the results in Table 1 for an electrically isolated droplet). The pulsed mode allows confirmation of this observation in a more controlled fashion because the electric field in the droplet area and production of corona ions can be controlled independently without rearrangement of the experimental apparatus. For this experiment, the laser firing time was set to $24 \mathrm{~ms}$ (in Period III), at which time a constant external electric field is established and the corona ions have been cleared out of the droplet area. The mass spectra were then collected for two instances: (1) with the application of $+4.5 \mathrm{kV}$ to the corona needle in Period I, resulting in production of corona ions; and (2) with the application of $+2.25 \mathrm{kV}$ to the corona needle, preventing corona ion formation in Period I. A significant loss in peptide ion signal is observed (similar to Periods II and IV in Figure 4), although desorption occurs in the presence of the constant external electric field established by the ring electrode in Period III. The same effect is observed when the corona needle is replaced with a blunt probe and a $+4.5 \mathrm{kV}$ potential is applied in Period I, again preventing corona ion formation.

To explain the observed effects of corona ions in signal generation and enhancement, we propose an alternative mechanism based on droplet-ion interactions. This mechanism is referred to as charge assisted laser desorption/ionization (CALDI) and differs from the standard liquid AP-MALDI in that the droplet is electrically isolated from the electrodes and bears a net charge due to the adsorption of corona ions onto the droplet surface (during Period I). When the charged droplet is placed in an external electric field (Periods I and III), the resulting asymmetric charge distribution in the droplet leads to an enhanced electric field on the side of the droplet closest to the MS inlet, facilitating ion formation during laser pulse. Similar effects for electrospray of droplets have been reported. For example, Grimm and Beauchamp reported that a charged droplet subjected to field induced droplet ionization (FIDI) deforms into an asymmetric (tear) shape in an external electric field, reducing the external field strength required to form gas-phase ions [23]. Similarly, coronainduced asymmetric electrospray of droplets near the tip of a corona needle has been attributed to charged droplets subjected to high electric fields at the tip of the needle [24, 25]. In addition, a model for the shape of the electric field around a charged spherical particle in an external electric field has been reported recently, demonstrating that the electric field is enhanced on one side of the particle as a result of asymmetric charge distribution. This enhancement is proportional to the amount of charge on the particle and depends inversely on the distance from the particle surface [26].

The FIDI and droplet electrospray experiments are performed at very high electric fields $\left(>1 \times 10^{6} \mathrm{~V} / \mathrm{m}\right)$ $[23,27]$. AP-MALDI also requires electric fields about $1 \times 10^{6} \mathrm{~V} / \mathrm{m}$ in the droplet area for optimum signal generation [28]. In contrast, much lower external electric fields are required for CALDI. The effects of external electric field on the analyte signals are compared in Figure 5 for CALDI and AP-MALDI of an electrically isolated droplet. For this experiment, pulsed mode 


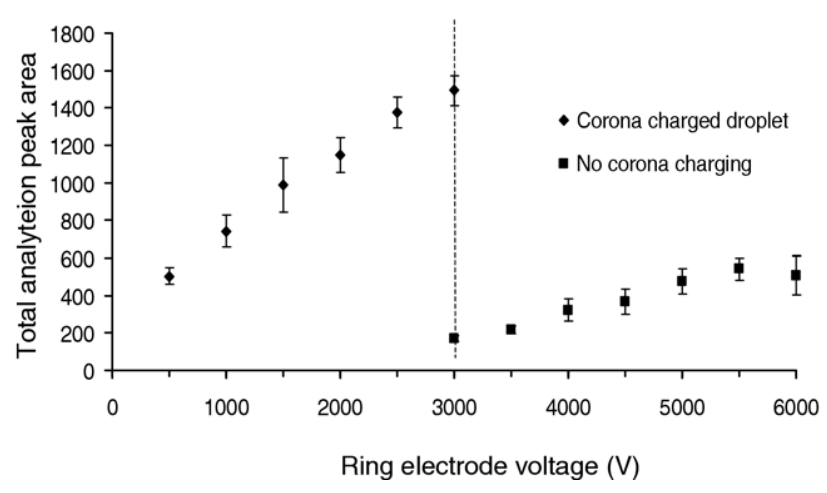

Figure 5. Effect of ring electrode voltage (external electric field) on ion generation from a charged droplet (CALDI) and an uncharged droplet (AP-MALDI) on a Teflon support.

CALDI was used with the same succession of events as depicted in Figure 3. Droplet charging occurred under constant conditions in Period I by applying $4.5 \mathrm{kV}$ to the voltage divider; and the laser pulse was fired at $24 \mathrm{~ms}$ (Period III). To vary the external electric field, the ring electrode voltage was adjusted from $500 \mathrm{~V}-3000 \mathrm{~V}$ in Period III, corresponding to $1 \times 10^{5} \mathrm{~V} / \mathrm{m}-6 \times 10^{5} \mathrm{~V} / \mathrm{m}$ at the droplet position based on SIMION (Scientific Instrument Services, Inc., Ringoes, NJ) simulations. The AP-MALDI experiments were performed in continuous mode where the corona needle was removed; a continuous voltage was applied to the ring electrode; and the laser was fired at $20 \mathrm{~Hz}$. Note that for the AP-MALDI signal to be observed in these conditions, the apex of the droplet rather than the closest surface to the nozzle had to be illuminated by the laser. This is also different from standard AP-MALDI where the sample is normally deposited on a stainless steel probe positioned in-line with the nozzle so that the apex of the droplet is the closest surface to the nozzle. Figure 5 shows clearly that the onset of ion generation is markedly different for charged and uncharged droplets. In CALDI, ion generation occurs at ring electrode voltages as low as $500 \mathrm{~V}$ $\left(\sim 1 \times 10^{5} \mathrm{~V} / \mathrm{m}\right)$, whereas no analyte ion signal could be detected below $3000 \mathrm{~V}\left(\sim 6 \times 10^{5} \mathrm{~V} / \mathrm{m}\right)$ for AP-MALDI. In addition, the dramatic improvement in sensitivity upon droplet charging is clearly shown in Figure 5. These results are in qualitative agreement with the enhanced electric field model at the droplet surface caused by the asymmetric charge distribution. Note that the analyte signal drops significantly for CALDI in the absence of an external electric field (Periods II and IV in Figure 4), indicating that the net charge on the droplet is not sufficient to create the required electric field on its own.

To investigate the stability of the charge on an electrically isolated droplet, a set of experiments were performed in which a $2-\mu \mathrm{L}$ droplet on a Teflon support was charged by 10 cycles of 5-ms corona ion bursts (similar to Period I in Figure 3). Ten cycles were used instead of one cycle to charge the droplet closer to the saturation point, thus minimizing the effect of charging variability in the experiments. A variable waiting period was then applied, before the droplet was analyzed by setting the ring electrode to $2.25 \mathrm{kV}$ and firing the laser at $20 \mathrm{~Hz}$. The mass spectra were collected at $1 \mathrm{spectrum} / \mathrm{s}$ until no signal was detected. The total analyte ion chromatograms for these experiments are illustrated in Figure 6 for wait periods of $5 \mathrm{~s}, 5 \mathrm{~min}$, and $15 \mathrm{~min}$. The peak areas are very similar and indicate that charge depletion due to events such as drainage through Teflon post is not significant on the time scale of this experiment.

\section{Further Mechanistic Considerations}

In previous sections, we concluded that droplet charging rather than gas-phase ion-neutral reactions is responsible for increased analyte ion signal in corona assisted UV-laser desorption. To further speculate on the mechanism of enhanced ion generation, two routes may be considered: (1) an AP-MALDI type mechanism and/or (2) desorption of charged clusters/droplets. These two routes are discussed below.

Laser irradiation of the sample in AP-MALDI creates both positive and negative ions, which are then separated and prevented from recombination (ion loss) by an external electric field [28]. An increased electric field at the surface of a charged droplet would result in a more effective and faster separation of the oppositely charged ions, reducing ion loss and thus improving the MS signal. Alternatively, the enhanced electric field and high charge density at the surface of the charged droplet could promote direct desorption of charged clusters/droplets upon laser irradiation which then evaporate and give birth to gaseous ions in an ESI-like mechanism. This mechanism is likely to be more pronounced in IR-MALDI where a greater extent of cluster/droplet desorption occurs compared to UVMALDI $[29,30]$. Enhancement of analyte ion signal has been observed in the presence of corona ions for APIR-MALDI and the effect has been attributed to neutral ionization $[5,6,31]$. However, no direct experimental evidence has been presented for this mechanism. In addition, it has recently been suggested that large

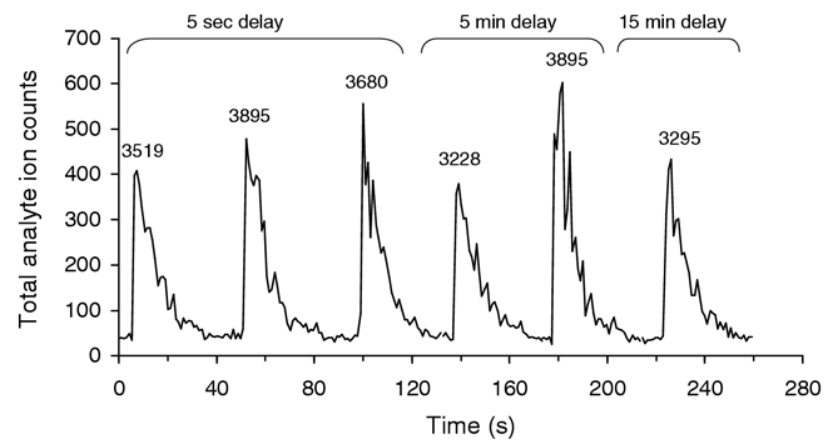

Figure 6. Total analyte ion chromatograms for a $2-\mu \mathrm{L}$ droplet (100 $\mu \mathrm{M}$ MRFA and bradykinin) on a Teflon support charged by 10 cycles of 5 -ms corona bursts. Spectra are collected at a 1 spectrum/s rate after the waiting period by setting the ring electrode to $2.25 \mathrm{kV}$ and firing the laser at $20 \mathrm{~Hz}$. The numbers above the peaks indicate the peak area. 
charged molecular clusters and/or small droplets desorbed in AP-IR-MALDI could be major contributors to the ion generation process via disintegration in the heated capillary of the MS inlet [31]. Accordingly, further charging of the clusters/droplets by corona ions could be a plausible mechanism for the observed signal enhancement in AP-IR-MALDI.

Regardless of the details of the CALDI mechanism discussed above, it is critical to note the differences between the LD-APCI and CALDI mechanisms, which affect their relative utility in various analytical applications. In the former, ionization occurs via a gas-phase proton transfer reaction between a corona-generated ion and a neutral analyte molecule. This reaction can be controlled thermodynamically by modification of the gas composition in the ionization area to provide a specific dominant ionization reagent. In contrast, in CALDI, corona ions are adsorbed onto the droplet surface, resulting in a net charge on the droplet. Therefore, the type of corona ion should have negligible effects on the efficiency and specificity of ionization in CALDI. Accordingly, different strategies will be required to improve the ionization efficiency for these two mechanisms.

\section{Conclusions and Future Directions}

The mechanism of signal enhancement for UV-APMALDI of liquid samples in the presence of positive corona ions was investigated. Our results indicate that for a droplet on a conducting support held at a high potential, analyte ion signal is enhanced in the presence of a corona needle largely because of the electric field modifications caused by the corona electrode, which increase the efficiency of ion transport into the MS. However, for an electrically isolated droplet, the analyte ion signal enhancement occurs as a consequence of droplet charging by corona ions rather than by gasphase reactions, hence, the term charge assisted laser desorption/ionization (CALDI). The excess charge on the droplet improves analyte ion formation by increasing the electric field at the droplet surface closest to the MS inlet upon application of an external electric field. Further studies of the CALDI process are underway, targeted towards understanding the role of two factors: (1) the gas-phase charge separation that occurs as a result of the larger electric field on the charged droplet and (2) the direct desorption of charged droplets/ clusters. We have demonstrated that the analytical performance of CALDI is similar to that of standard liquid AP-MALDI. Note that sensitivity in CALDI is proportional to the laser repetition rate, and higher repetition rates will provide higher sensitivities. Additional gains in sensitivity can be achieved by increasing the duty cycle via synchronizing the laser desorption with the TOF orthogonal extraction. In CALDI, direct electrical contact with the sample is eliminated and gas-phase ions can be generated at about one order of magnitude lower external electric fields compared to standard AP-MALDI and ESI of droplets. This simpli- fies the instrumentation, especially in situations where the electrodes cannot be placed in close proximity to the sample to generate a high electric field. One example is from recent studies from our laboratory on generating gas-phase ions from acoustically levitated droplets, where objects placed in close proximity to the droplet would disturb the levitation field and destabilize the droplet levitation [32]. The results presented in the current report clearly demonstrate that droplet charging plays an important role when corona ions are used to improve the ionization efficiency of laser desorption/ionization techniques, and that the gas-phase ion-neutral reactions which are central to the mechanism of APCI may not be the dominant ionization mechanism in LD-APCI.

\section{Acknowledgments}

The authors express their gratitude to the National Heart, Lung, and Blood Institute for financial support under National Proteomics Center contract number N01-HV-28,182.

\section{References}

1. Fenn, J. B. M.; Meng, C. K.; Wong, S. F.; Whitehouse, C. M. Electrospray Ionization for Mass Spectrometry of Large Biomolecules. Science 1989, 246, 64-71

2. Karas, M.; Hillenkamp, F. Laser Desorption Ionization of Proteins with Molecular Masses Exceeding 10,000 Daltons. Anal. Chem. 1988, 60, 2299-2301.

3. Takats, Z.; Wiseman, J. M.; Gologan, B.; Cooks, R. G. Mass Spectrometry Sampling Under Ambient Conditions with Desorption Electrospray Ionization. Science 2004, 306, 471-473.

4. Robb, D. B.; Covey, T. R.; Bruins, A. P. Atmospheric Pressure Photoionization: An Ionization Method for Liquid Chromatography-Mass Spectrometry. Anal. Chem. 2000, 72, 3653-3659.

5. Coon, J. J.; McHale, K. J.; Harrison, W. W. Atmospheric Pressure Laser Desorption/Chemical Ionization Mass Spectrometry: A New Ionization Method Based on Existing Themes. Rapid Commun. Mass Spectrom. 2002, $16,681-685$.

6. Coon, J. J.; Harrison, W. W. Laser Desorption-Atmospheric Pressure Chemical Ionization Mass Spectrometry for the Analysis of Peptides from Aqueous Solutions. Anal. Chem. 2002, 74, 5600-5605.

7. Turney, K.; Harrison, W. W. Corona Discharge Secondary Ionization of Laser Desorbed Neutral Molecules from a Liquid Matrix at Atmospheric Pressure. Spectrochim. Acta 2006, 61B, 634-641.

8. Shiea, J.; Huang, M.-Z.; Hsu, H.-J.; Lee, C.-Y.; Yuan, C.-H.; Beech, I.; Sunner, J. Electrospray-Assisted Laser Desorption/Ionization Mass Spectrometry for Direct Ambient Analysis of Solids. Rapid Commun. Mass Spectrom. 2005, 19, 3701-3704.

9. Huang, M.-Z.; Hsu, H.-J.; Wu, C.-I.; Lin, S.-Y.; Ma, Y.-L.; Cheng, T.-L.; Shiea, J. Characterization of the Chemical Components on the Surface of Different Solids with Electrospray-Assisted Laser Desorption Ionization Mass Spectrometry. Rapid Commun. Mass Spectrom. 2007, 21, 1767-1775.

10. Nemes, P.; Vertes A. Laser Ablation Electrospray Ionization for Atmospheric Pressure, in Vivo, and Imaging Mass Spectrometry. Anal. Chem. 2007, 79, 8098-8106.

11. Cristoni, S.; Bernardi, R. S.; Biunno, I.; Guidulgi, F. Analysis of Protein Ions in the Range 3000-12000 Th Under Partial (No Discharge) Atmospheric Pressure Chemical Ionization Conditions Using Ion Trap Mass Spectrometry. Rapid Commun. Mass Spectrom. 2002, 16, 1153-1159.

12. Cristoni, S.; Bernardi, R. S.; Biunno, I.; Guidulgi, F. Analysis of Protein Peptides Using Partial (No Discharge) Atmospheric Pressure Chemical Ionization Conditions with Ion Trap Mass Spectrometry. Rapid Commun. Mass Spectrom. 2002, 16, 1686-1691.

13. Cody, R. B.; Laramee, J. A.; Durst, H. D. Versatile New Ion Source for the Analysis of Materials in Open Air Under Ambient Conditions. Anal. Chem. 2005, 77, 2297-2302.

14. Takáts, Z.; Cotte-Rodriguez, I.; Talaty, N.; Chen, H.; Cooks, R. G. Direct Trace Level Detection of Explosives on Ambient Surfaces by Desorption Electrospray Ionization Mass Spectrometry. Chem. Commun. 2005, 15, 1950-1952.

15. Golovlev, V. V.; Allman, S. L.; Garrett, W. R.; Taraneko, N. I.; Chen, C. H. Laser Induced Acoustic Desorption. Int. J. Mass Spectrom. Ion Processes 1997, 169/170, 69-78.

16. Shea, R. C.; Petzold, C. J.; Liu, J. A.; Kenttamaa, H. I. Experimental Investigations of the Internal Energy of Molecules Evaporated Via Laser-Induced Acoustic Desorption into a Fourier Transform Ion Cyclotron Resonance Mass Spectrometer. Anal. Chem. 2007, 79, 1825-1832. 
17. Grimm, R. L; Beauchamp, J. L. Field-Induced Ddroplet Ionization Mass Spectrometry. J. Phys. Chem. B 2003, 107, 14161-14163.

18. Northen, T. R.; Yanes, O.; Northen, M. T.; Marrinucci, D.; Uritboonthai, W.; Apon, J.; Golledge, S. L.; Nordström1, A.; Siuzdak, G. Clathrate Nanostructures for Mass Spectrometry. Nature 2007, 449, 1033-1037.

19. Sze, E. T. P.; Chan, T.-W. D. Formulation of Matrix Solutions for Use in Matrix Assisted Laser Desorption/Ionization of Biomolecules. J. Am. Soc. Mass Spectrom. 1998, 9, 166-174.

20. Armstrong, D. W.; Zhang, L.-K.; He, L.; Gross, M. L. Ionic Liquids as Matrixes for Matrix-Assisted Laser Desorption/Ionization Mass Spectrometry. Anal. Chem. 2001, 73, 3679-3686.

21. Li, Y. L.; Gross, M. L. Ionic-Liquid Matrices for Quantitative Analysis by MALDI-TOF Mass Spectrometry. J. Am. Soc. Mass Spectrom. 2004, 15, 1833-1837.

22. Turney, K.; Harrison, W. W. Liquid Supports for Ultraviolet Atmospheric Pressure Matrix-Assisted Laser Desorption/Ionization. Rapid Commun. Mass Spectrom. 2004, 18, 629-635.

23. Grimm, R. L.; Beauchamp, J. L. Dynamics of Field-Induced Droplet Ionization: Time-Resolved Studies of Distortion, Jetting, and Progeny Formation from Charged and Neutral Methanol Droplets Exposed to Strong Electric Fields. J. Phys. Chem. B 2005, 109, 8244-8250.

24. Hager, D. B.; Dovichi, N. J. Behavior of Microscopic Liquid Droplets Near a Strong Electrostatic Field: Droplet Electrospray. Anal. Chem. 1994, 66, 1593-1594.
25. Hager, D. B.; Dovichi, N. J.; Klassen, J.; Kebarle, P. Droplet Electrospray Mass Spectrometry. Anal. Chem. 1994, 66, 3944-3949.

26. Adamiak, K. Rate of Charging of Spherical Particles by Monopolar Ions in Electric Fields. IEEE Trans. Ind. Applicat. 2002, 38, 1001-1008.

27. Grimm, R. L.; Hodyss, R.; Beauchamp, J. L. Probing Interfacial Chemistry of Single Droplets with Field-Induced Droplet Ionization Mass Spectrometry: Physical Adsorption of Polycyclic Aromatic Hydrocarbons and Ozonolysis of Oleic Acid and Related Compounds. Anal. Chem. 2006, 78, 3800-3806.

28. Berkout, V. D.; Kryuchkov, S. I.; Doroshenko, V. M. Modeling of Ion Processes in Atmospheric Pressure Matrix-Assisted Laser Desorption/ Ionization. Rapid Commun. Mass Spectrom. 2007, 21, 2046-2050.

29. Jackson, S. N.; Kim, J.-K.; Laboy, J. L.; Murray, K. K. Particle Formation by Infrared Laser Ablation of Glycerol: Implication for Ion Formation. Rapid Commun. Mass Spectrom. 2006, 20, 1299-1304.

30. Leisner, A.; Rohlfing, A.; Röhling, U.; Dreisewerd, K.; Hillenkamp, F. Time-Resolved Imaging of the Plume Dynamics in Infrared MatrixAssisted Laser Desorption/Ionization with a Glycerol Matrix. J. Phys. Chem. B 2005, 109, 11661-11666.

31. Laiko, V. V.; Taranenko, N. I.; Doroshenko, V. M. On the Mechanism of Ion Formation from the Aqueous Solutions Irradiated with $3 \mu \mathrm{m}$ IR Laser Pulses Under Atmospheric Pressure. J. Mass Spectrom. 2006, 41 1315-1321.

32. Westphall, M. S.; Jorabchi, K.; Smith, L. M. Mass Spectrometry of Acoustically Levitated Droplets. Unpublished. 